\title{
Preservation of renal function by intensive glycemic control
}

\author{
Naoya Toriu', Masayuki Yamanouchi', Rikako Hiramatsu', Noriko Hayami', Junichi Hoshino', Akinari Sekine1, \\ Masahiro Kawada1, Eiko Hasegawa', Tatsuya Suwabe1, Keiichi Sumida1, Toshiharu Ueno', Naoki Sawa', \\ Kenichi Ohashi2,4, Takeshi Fujii', Kenmei Takaichi1,3, Motoko Yanagita5, \\ Tetsuro Kobayasi ${ }^{3}$ and Yoshifumi Ubara1,3 \\ ${ }^{1}$ Nephrology Center and Department of Rheumatology, Toranomon Hospital, Tokyo, Japan, ${ }^{2}$ Department of \\ Pathology, Toranomon Hospital, Tokyo, Japan, ${ }^{3}$ Okinaka Memorial Institute for Medical Research, Tokyo, Japan, \\ ${ }^{4}$ Department of Pathology, Yokohama City University, Graduate School of Medicine, Yokohama, Japan, and \\ ${ }^{5}$ Department of Nephrology, Kyoto University Graduate School of Medicine, Japan

\section{Correspondence} \\ should be addressed \\ to $\mathrm{N}$ Toriu or Y Ubara \\ Email \\ torippy.naoya@gmail.com or \\ ubara@toranomon.gr.jp
}

\section{Summary}

We report the case of a 67-year-old Japanese woman with type 1 diabetes mellitus. At 47 years of age, her hemoglobin A1c (HbA1c) was $10.0 \%$, and she had overt nephropathy. The first renal biopsy yielded a diagnosis of diabetic nephropathy. Intensive glycemic control was initiated and her HbA1c improved to $6.0 \%$. Renal dysfunction showed no progression for 15 years. At 62 years of age, a second renal biopsy was performed. Glomerular lesions did not show progression but tubulointerstitial fibrosis and vascular lesions showed progression compared with the first biopsy. Intensive glycemic control can prevent the progression of glomerular lesions, but might not be effective for interstitial and vascular lesions.

\section{Learning points:}

- Intensive control of blood glucose can prevent the progression of glomerular lesions.

- Intensive control of blood glucose may not be able to prevent progression of interstitial and vascular lesions.

- CSII reduces $\mathrm{HbA1c}$ without increasing the risk of hypoglycemia.

\section{Background}

Diabetic nephropathy (DN) is one of the most important causes of end-stage renal disease (ESRD). Hyperglycemia is a necessary precondition for development of $\mathrm{DN}$, which features an increase of the mesangial matrix and hyalinosis of glomerular arteries (1). Tervaert and coworkers developed a consensus classification of DN for both type 1 and type 2 diabetes mellitus, which divides patients into four classes based on glomerular lesions (class I, class II (a and b), class III and class IV) (2). In addition, DN is classified into stages 1-5 based upon the estimated glomerular filtration rate (eGFR) and urinary protein excretion and/or albuminuria. In stages $1-3$ of $\mathrm{DN}$, eGFR is $\geq 30 \mathrm{~mL} / \mathrm{min} / 1.73 \mathrm{~m}^{2}$, while eGFR is
$<30 \mathrm{~mL} / \mathrm{min} / 1.73 \mathrm{~m}^{2}$ in stages 4 and 5 . Stages $1-3$ are separated on the basis of urinary albumin or protein excretion, as follows. Stage 1 is called prenephropathy and is defined by urinary albumin excretion of $\leq 30 \mathrm{mg} / \mathrm{gCr}$, while Stage 2 is called incipient nephropathy and is defined by urinary albumin excretion of $>30 \mathrm{mg} / \mathrm{gCr}$ and $\leq 300 \mathrm{mg} / \mathrm{gCr}$. Stage 3 is called overt nephropathy and is defined as urinary albumin excretion $>300 \mathrm{mg} / \mathrm{gCr}$ or urinary protein excretion $>0.5 \mathrm{~g} / \mathrm{gCr}$ (3).

It is known that intensive glycemic control improves prenephropathy (stage 1) and incipient nephropathy (stage 2) (4), but there is no consensus about its effect on overt nephropathy (stage 3 ). Moreover, the effect of 
Table 1 Laboratory findings.

\begin{tabular}{l}
\hline \\
\hline Blood pressure $(\mathrm{mmHg})$ \\
Hemoglobin $(\mathrm{mg} / \mathrm{dL})$ \\
Urea nitrogen $(\mathrm{mg} / \mathrm{dL})$ \\
Creatinine $(\mathrm{mg} / \mathrm{dL})$ \\
eGFR $\left(\mathrm{mL} / \mathrm{min} / 1.73 \mathrm{~m}^{2}\right)$ \\
HbA1c $(\%)$ \\
Urinary albumin excretion $(\mathrm{mg} / \mathrm{g} \mathrm{Cr})$ \\
Urinary protein $(\mathrm{g} /$ day) \\
\hline
\end{tabular}

\begin{tabular}{c|}
\hline T1DM diagnosed \\
\hline 1989 \\
\hline $128 / 82$ \\
10.0 \\
14 \\
0.7 \\
87 \\
11.0 \\
29.4 \\
NA
\end{tabular}

\begin{tabular}{|c|c|c|}
\hline \multicolumn{3}{|c|}{ Insulin pump } \\
\hline 1996 (1st RB) & 2011 & 2013 (2nd RB) \\
\hline $116 / 70$ & $140 / 84$ & $157 / 88$ \\
\hline 10.7 & 15.0 & 14.4 \\
\hline 14 & 24 & 28 \\
\hline 1.0 & 1.0 & 1.22 \\
\hline 35.9 & 44.0 & 35.1 \\
\hline 8.9 & 6.4 & 6.8 \\
\hline NA & NA & NA \\
\hline 3.05 & 1.14 & 2.21 \\
\hline
\end{tabular}

\begin{tabular}{c}
\hline HD \\
\hline 2017 \\
\hline $141 / 63$ \\
11.9 \\
117 \\
8.83 \\
4 \\
5.7 \\
NA \\
8.89 \\
\hline
\end{tabular}

\begin{tabular}{c}
\hline Normal range \\
\hline$<130 / 80$ \\
$11.3-15.0$ \\
$8-12$ \\
$0.65-1.06$ \\
$>90$ \\
$<6.5$ \\
$<30$ \\
$<0.1$
\end{tabular}

$\mathrm{RB}$, renal biopsy.

intensive glycemic control on progression of renal lesions has not been reported.

We report a patient with type 1 diabetes mellitus (T1DM) and overt diabetic nephropathy in whom progression of renal dysfunction and glomerular lesions was prevented for 15 years by intensive glycemic control using an insulin pump.

\section{Case presentation}

In 1980, at the age of 30 years, a Japanese woman was diagnosed with T1DM. Her mean hemoglobin A1c (HbA1c) was approximately $10 \%$ on insulin therapy, although her blood pressure was controlled well by enalapril $10 \mathrm{mg}$. In 1996, at the age of 47 years, serum creatinine was increased from $0.7 \mathrm{mg} / \mathrm{dL}$ to $1.0 \mathrm{mg} / \mathrm{dL}$ and proteinuria was increased to $3 \mathrm{~g} /$ day.

\section{Investigation}

At that time, the patient was $158 \mathrm{~cm}$ tall and weighed $54.6 \mathrm{~kg}$, with a blood pressure of $116 / 70 \mathrm{mmHg}$ and a temperature of $36.7^{\circ} \mathrm{C}$. Laboratory findings (Table 1) were as follows: white blood cell count: $6000 / \mu \mathrm{L}$; red blood cell count: $3.92 \times 10^{4} / \mu \mathrm{L}$; hemoglobin: $10.7 \mathrm{~g} / \mathrm{dL}$; total protein: $6.3 \mathrm{~g} / \mathrm{dL}$; albumin: $3.6 \mathrm{~g} / \mathrm{dL}$; urea nitrogen: $13 \mathrm{mg} / \mathrm{dL}$; serum creatinine: $1.0 \mathrm{mg} / \mathrm{dL}$; eGFR: $35.9 \mathrm{~mL} / \mathrm{min}$, total cholesterol: $211 \mathrm{mg} / \mathrm{dL}$; CRP: $0.0 \mathrm{mg} / \mathrm{dL}$ and HbA1c: $9.2 \%$. Her 24-h urine protein excretion was $3.05 \mathrm{~g}$. Moderate non-proliferative diabetic retinopathy (NPDR) was noted. The first renal biopsy was performed.

\section{First renal biopsy}

Light microscopic examination of the renal biopsy specimen revealed global sclerosis in 2 of 10 glomeruli. The preserved glomeruli showed an increase of mesangial matrix with mesangiolysis, but mesangial expansion was smaller than the mean capillary lumen area in $>25 \%$ of the preserved glomeruli and nodular lesions were not observed. Immunofluorescence (IF) demonstrated linear deposition of immunoglobulin $\mathrm{G}$ (IgG) along the capillary walls. Electron microscopy (EM) revealed that the average glomerular basement membrane (GBM) thickness was greater than $395 \mathrm{~nm}$. Class IIA DN was diagnosed according to Tervaert's classification (2). Interstitial fibrosis and tubular atrophy (IFTA) occupied less than $25 \%$ of the total renal cortical area and interstitial inflammation was only seen around atrophic tubules. Arteriolar hyalinosis was absent, and interlobular arteries only showed mild arteriosclerosis with the thickness of the intima being less than that of the media. The score of interstitial and vascular lesions of DN according to Tervaert's classification (2) was as follows: IFTA, 1; interstitial inflammation, 1 ; arteriolar

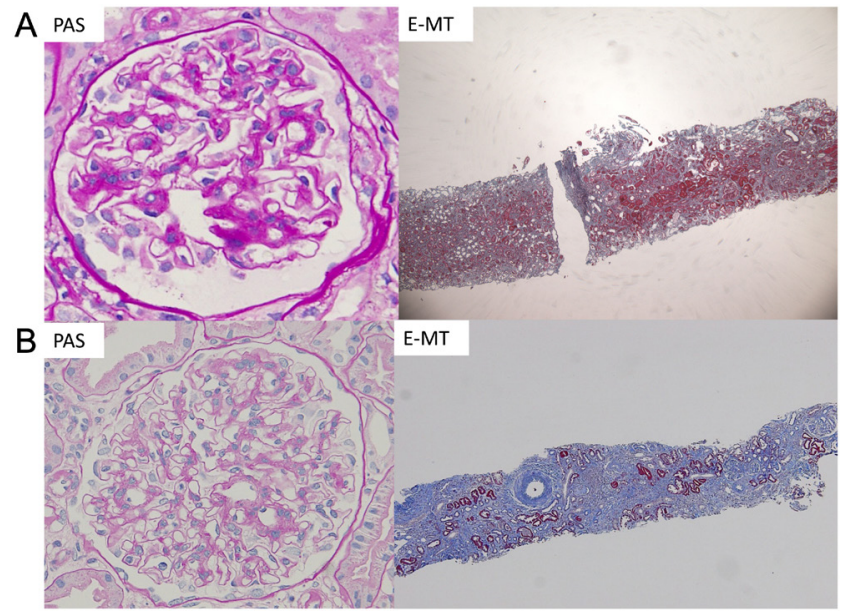

Figure 1

Renal biopsy findings. (A) First biopsy: light microscopy displays mesangial proliferation with mesangiolysis and mild tubulointerstital fibrosis. (B) Second biopsy: light microscopy reveals mesangial proliferation with mesangiolysis and moderate tubulointerstital fibrosis. E-MT, Elastica Masson trichrome stain; PAM, Periodic acid methenamine-silver stain; PAS: Periodic acid-Schiff stain. 
hyalinosis, 0 and arteriosclerosis was 1 . The total score for interstitial and vascular lesions of DN was 3 (Fig. 1A).

\section{Treatment}

\section{Clinical course}

Continuous subcutaneous insulin infusion (CSII) was initiated with an insulin pump and her HbA1c improved to $6 \%$, while the frequency of hypoglycemic episodes was not increased. Serum creatinine decreased to $0.8 \mathrm{mg} / \mathrm{dL}$ and proteinuria decreased to $1 \mathrm{~g} /$ day. Subsequently, there was no progression of renal dysfunction for 15 years. However, renal function began to decline from 2011 and proteinuria increased to $2 \mathrm{~g}$ /day in July 2013, so a second renal biopsy was performed.

At the time of the second biopsy, the patient weighed $52.5 \mathrm{~kg}$, with a blood pressure of $157 / 88 \mathrm{mmHg}$ and a temperature of $36.7^{\circ} \mathrm{C}$. Laboratory findings (Table 1 ) were as follows: white blood cell count: $6400 / \mu \mathrm{L}$; red blood cell count: $4.85 \times 10^{4} / \mu \mathrm{L}$; hemoglobin: $14.4 \mathrm{~g} / \mathrm{dL}$; total protein: $7.3 \mathrm{~g} / \mathrm{dL}$; albumin: $4.1 \mathrm{~g} / \mathrm{dL}$; urea nitrogen: $28 \mathrm{mg} / \mathrm{dL}$; serum creatinine: $1.22 \mathrm{mg} / \mathrm{dL}$; eGFR: $35.1 \mathrm{~mL} / \mathrm{min}$, total cholesterol: $231 \mathrm{mg} / \mathrm{dL}$; CRP: $0.0 \mathrm{mg} / \mathrm{dL}$; HbA1c: $6.8 \%$ and glycoalbumin: $16.7 \%$. Her 24-h urine protein excretion was $2.21 \mathrm{~g}$. Funduscopy revealed the same moderate NPDR as that seen 17 years earlier.

\section{Outcome and follow-up}

\section{Second renal biopsy}

On light microscopy of the renal biopsy specimen, global sclerosis was detected in 6 of 23 glomeruli. Mesangial expansion did not exceed the mean capillary lumen area in $>25 \%$ of the preserved glomeruli, and there was no nodular sclerosis, so DN was class IIa according to Tervaert's classification. On the other hand, interstitial fibrosis and tubular atrophy occupied more than 50\% of the total renal cortical area and interstitial inflammation was noted around atrophic tubules. In addition, more than one arteriole showed hyalinosis in the entire biopsy specimen and the interlobular arteries displayed mild arteriosclerosis with the thickness of the intima exceeding that of the media. The score of interstitial and vascular lesions of DN according to Tervaert's classification (2) was as follows; IFTA, 2; interstitial inflammation, 1; arteriolar hyalinosis, 2 and arteriosclerosis was 2 . Thus, the total score for interstitial and vascular lesions of DN was 7. Both interstitial and vascular lesions showed progression compared with the first biopsy, although glomerular

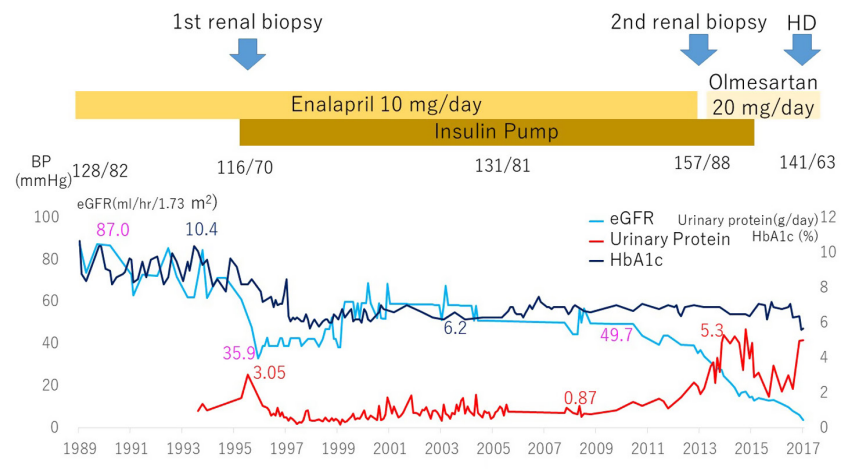

Figure 2

Clinical course.

lesions were unchanged. IF and EM findings were the same as before (Fig. 1B).

\section{Clinical course}

Intensive glycemic control was continued thereafter. Before the second renal biopsy, blood pressure was controlled by only enalapril; however, after the second renal biopsy, blood pressure control was difficult so enalapril was changed to olmesartan, but her renal function continued to deteriorate. In April 2017, at the age of 67 years, hemodialysis was started after serum creatinine reached $8.8 \mathrm{mg} / \mathrm{dL}$, and she developed congestive heart failure (Fig. 2).

\section{Discussion}

In the present, T1DM patient with overt DN, marked progression of renal dysfunction and glomerular lesions was prevented for 15 years by intensive glycemic control using CSII.

The Diabetes Control and Complications Trial research group (DCCT) demonstrated that T1DM patients with normoalbuminuria or microalbuminuria achieved improvement or maintenance of renal function on intensive glycemic control (4). However, there have been no randomized studies investigating the influence of glycemic control on renal function in T1DM patients with macroalbuminuria. The American Diabetes Association emphasizes that the HbA1c target should be individualized for each patient and that improved glycemic control prevents microvascular complications, suggesting a reasonable $\mathrm{HbA} 1 \mathrm{c}$ target of $<7 \%$ for adults with diabetes or $<8.5 \%$ for patients with advanced microvascular or macrovascular complications (5).

Improving the control of blood glucose may prevent deterioration of renal function. A retrospective cohort 
study showed that reducing $\mathrm{HbA} 1 \mathrm{c}$ from $10 \%$ to $8 \%$ could decrease the cumulative risk of developing ESRD after 15 years for T1DM patients with overt albuminuria to $29 \%$ compared with $45 \%$ for standard therapy (6). Moreover, normalizing blood glucose may prevent further progression of renal lesions or allow recovery. In T1DM patients who received renal allografts, intensive therapy prevented worsening of glomerular lesions after 5 years, unlike standard therapy (7), while T1DM patients undergoing pancreatic transplantation showed improvement of glomerular lesions after 10 years of follow-up (1). In other T1DM patients undergoing pancreatic transplantation, tubulointerstital lesions showed progression at the 5 -year assessment because of cyclosporine therapy, but recovered at the 10-year assessment because of a lower cyclosporine dose or prolonged normoglycemia (8). In our patient, intensive glycemic control prevented the progression of glomerular lesions, but tubulointerstital lesions still became more advanced. Arteriolar sclerosis and arteriolar hyalinosis were advanced, so interstitial lesions might be caused by ischemia. This may have been because intensive control with CSII does not completely normalize the blood glucose level or because the patient's blood pressure was not controlled sufficiently.

Generally, both glomerular lesions and interstitial lesions are progressed in DMN patients so this case seems to be peculiar; however, there has been no report focusing on the long-term effect of intensive glycemic control on glomerular lesions and interstitial lesions without transplantation so further studies are required.

On the other hand, intensive glycemic control increases the risk of hypoglycemia. In the DCCT trial, there were 62 hypoglycemic episodes per 100 patient-years in the intensive therapy group vs only 19 hypoglycemic episodes per 100 patient-years in the standard therapy group (4). Hypoglycemic episodes increase the risk of mortality and cardiovascular disease (CVD). In a Taiwanese cohort of 10,411 patients with T1DM, hypoglycemia increased the frequency of all-cause mortality and CVD (with an adjusted odds ratio of 2.74 and 2.02, respectively) (9).

CSII reduces the HbA1c without increasing the risk of hypoglycemia. In a randomized, open-label, cross-over study performed by Hirsch, CSII improved the control of blood glucose without increasing the risk of hypoglycemia compared to multiple daily insulin injections (10).

In conclusion, a T1DM patient developed overt nephropathy and showed a rapid decrease of eGFR during the 16-year period after diagnosis. However, after intensive glycemic control was initiated using CS11, renal function did not worsen for the next 15 years, and there was no increase of hypoglycemic episodes. This case indicates that intensive control of blood glucose can prevent the progression of glomerular lesions, but may not be able to prevent progression of interstitial and vascular lesions. Exacerbation of interstitial lesions may have contributed to the decline of renal dysfunction in this patient during the last 4 years before hemodialysis (Table 1).

\section{Declaration of interest}

The authors declare that there is no conflict of interest that could be perceived as prejudicing the impartiality of the research reported.

\section{Funding}

This study was funded by the Okinaka Memorial Institute for Medical Research.

\section{Patient consent}

The present report adhered to the Declaration of Helsinki, and the patient gave his consent for the case report to be published.

\section{Author contribution statement}

Naoya Toriu: writing; Masayuki Yamanouchi: study design; Rikako Hiramatsu: data interpretation; Noriko Hayami: data analysis; Junichi Hoshino: surgery; Akinari Sekine: data analysis; Masahiro Kawada: surgery; Eiko Hasegawa: data interpretation; Tatsuya Suwabe: surgery; Keiichi Sumida: data analysis; Toshiharu Ueno: data analysis; Naoki Sawa: study design; Kenichi Ohashi: surgery; Takeshi Fujii: surgery; Kenmei Takaichi: data collection; Motoko Yanagita: data analysis; Tetsuro Kobayashi: analysis MD and Yoshifumi Ubara: writing and generalization.

\section{References}

1 Fioretto P, Steffes MW, Sutherland DE, Goetz FC \& Mauer M. Reversal of lesions of diabetic nephropathy after pancreas transplantation. New England Journal of Medicine 1998339 69-75. (https://doi.org/10.1056/NEJM199807093390202)

2 Tervaert TW, Mooyaart AL, Amann K, Cohen AH, Cook HT, Drachenberg CB, Ferrario F, Fogo AB, Haas M, de Heer E, et al. Pathologic classification of diabetic nephropathy. Journal of the American Society of Nephrology 201021 556-563. (https://doi. org/10.1681/ASN.2010010010)

3 Haneda M, Utsunomiya K, Koya D, Babazono T, Moriya T, Makino H, Kimura K, Suzuki Y, Wada T, Ogawa S, et al. A new classification of diabetic nephropathy 2014: a report from Joint Committee on Diabetic Nephropathy. Journal of Diabetes Investigation 20156 242-246. (https://doi.org/10.1111/jdi.12319)

4 Nathan DM, Genuth S,

Lachin JClearyPCroffordODavisMRandLSiebertC. The effect of intensive treatment of diabetes on the development and progression of long-term complications in insulin-dependent diabetes mellitus. New England Journal of Medicine 1993329 977-986. (https://doi. org/10.1056/NEJM199309303291401) 
5 Chiang JL, Kirkman MS, Laffel LM \& Peters AL. Type 1 diabetes sourcebook A. Type 1 diabetes through the life span: a position statement of the American Diabetes Association. Diabetes Care 2014 37 2034-2054. (https://doi.org/10.2337/dc14-1140)

6 Skupien J, Warram JH, Smiles A, Galecki A, Stanton RC \& Krolewski AS. Improved glycemic control and risk of ESRD in patients with type 1 diabetes and proteinuria. Journal of the American Society of Nephrology 201425 2916-2925. (https://doi.org/10.1681/ ASN.2013091002)

7 Barbosa J, Steffes MW, Sutherland DE, Connett JE \& Rao KV, Mauer SM. Effect of glycemic control on early diabetic renal lesions. A 5-year randomized controlled clinical trial of insulin-dependent diabetic kidney transplant recipients. JAMA 1994272 600-606. (https://doi.org/10.1001/jama.1994.03520080042041)
8 Mauer M \& Fioretto P. Pancreas transplantation and reversal of diabetic nephropathy lesions. Medical Clinics of North America 2013 97 109-114. (https://doi.org/10.1016/j.mcna.2012.10.009)

$9 \mathrm{Lu}$ CL, Shen HN, Hu SC, Wang JD \& Li CY. A population-based study of all-cause mortality and cardiovascular disease in association with prior history of hypoglycemia among patients with type 1 diabetes. Diabetes Care 201639 1571-1578. (https://doi.org/10.2337/dc15-2418)

10 Hirsch IB, Bode BW, Garg S, Lane WS, Sussman A, Hu P, Santiago OM, Kolaczynski JW \& Insulin Aspart CSII/MDI Comparison Study Group. Continuous subcutaneous insulin infusion (CSII) of insulin aspart versus multiple daily injection of insulin aspart/insulin glargine in type 1 diabetic patients previously treated with CSII. Diabetes Care 200528 533-538. (https://doi. org/10.2337/diacare.28.3.533)

Received in final form 20 November 2017 Accepted 6 December 2017 